\title{
First trial with an all-fibre near infrared spectrometer evaluated by multivariate curve resolution
}

\author{
T. Ringsted, ${ }^{a *}$ B. Robotham, ${ }^{b}$ J.D. Buron, ${ }^{b}$ K.M. Sørensen, ${ }^{a}$ T. Nikolajsen ${ }^{b}$ and S.B. Engelsen ${ }^{a}$ \\ aSpectroscopy \& Chemometrics, Department of Food Science, University of Copenhagen, Frederiksberg, Denmark. E-mail: tine.ringsted@food.ku.dk, \\ kms@food.ku.dk, se@foof.ku.dk \\ boss Analytical A/S, Foss Allé 1, 3400 Hillerød, Denmark. E-mail: bero@foss.dk, jbu@foss.dk, tni@foss.dk
}

\begin{abstract}
A novel all-fibre spectrometer with theoretical advantages for industrial process measurements was tested on simulated process samples of water with sucrose. The spectrometer uses the dispersive Fourier transform method and combines a supercontinuum laser with a time-wavelength separating optical fibre. Multivariate curve resolution was used to resolve the pure spectra and concentration profiles of sucrose and water from spectra of solutions with both constituents. The new spectrometer has great potential for rapid and vibration-robust measurements, but further studies are needed to determine its performance on more complex and real process samples.
\end{abstract}

\section{Introduction}

Rapid and robust multivariate measurements are some of the most desired properties for process instruments. A novel spectroscopic method named "dispersive Fourier transform" has recently been pushing the limit of measurement speed. ${ }^{1,2}$ The principle behind dispersive Fourier transform is a polychromatic pulsed light source that is directed through an element that separates the different wavelengths in exit time. The signal can then be transformed from the timedomain into the frequency domain to provide a spectrum. A typical element used to separate the wavelengths in time is an optical fibre. The applied light source can be a pulsed supercontinuum, which is commonly produced by guiding intense light through photonic crystal fibres. ${ }^{3}$ An "all-fibre spectrometer" can, therefore, be constructed by combining a dispersive optical fibre with a supercontinuum fibre laser resulting in an instrument with no moving parts that is insensitive to vibrations and has the potential for extremely rapid pulsed measurements. Currently there are no commercial spectrometers applying the dispersive Fourier transform method and the theoretical advantages have, therefore, not been studied and exploited in an industrial context.

The measurement of carbohydrates in the food industry is important in terms of analysing product variations as well as following processes such as, for example, fermentations. The common food ingredient "table sugar" or sucrose ( $\beta$-D-fructofuranosyl-a-D-glucopyranoside) is a central component in a wide range of food products, and near infrared (NIR) spectroscopy has been used in several cases to measure the sucrose content such as, for instance, in sugar beets, ${ }^{4}$ coffee roasting ${ }^{5}$ and sugar cane juice for the alcohol industry. ${ }^{6}$ In the present study, the performance of an in-house built dispersive Fourier transform NIR spectrom eter was tested on mixtures of water with sucrose. This was performed in order to test the spectral quality of a simulated industrial process measured with a dispersive Fourier transform spectrometer. The resulting spectra were analysed with Principal Component Analysis (PCA) to visualise the spectral changes on a large range of sucrose concentrations. In addition, Multivariate Curve Resolution (MCR) was applied in order to investigate the quality of the NIR spectra and to

\section{Correspondence}

T. Ringsted (tine.ringsted@food.ku.dk)

doi: $10.1255 /$ nir2017.169

Citation: T. Ringsted, B. Robotham, J.D. Buron, K.M. Sørensen, T. Nikolajsen

and S.B. Engelsen, "First trial with an all-fibre near infrared spectrometer

evaluated by multivariate curve resolution", in Proc. $18^{\text {th }}$ Int. Conf. Near

Infrared Spectrosc., Ed by S.B. Engelsen, K.M. Sørensen and F. van den

Berg. IM Publications Open, Chichester, pp. 169-175 (2019). https://doi.

org/10.1255/nir2017.169

\section{(c) 2019 The Authors}

This licence permits you to use, share, copy and redistribute the paper in any medium or any format provided that a full citation to the original paper is given.

ISBN: 978-1-906715-27-4 
demonstrate separation of the pure spectrum of water from the pure spectrum of sucrose, as MCR in previous studies has shown a large potential in process monitoring of milk lactic acid fermentation ${ }^{7}$ and alcohol fermentation measured with NIR spectroscopy. . $^{8}$

\section{Methods}

\section{Samples}

One sample of Milli-Q ${ }^{\circledR}$ water and 51 samples of Milli-Q ${ }^{\circledR}$ water with sucrose from $5 \%$ to $55 \%(\mathrm{w} / \mathrm{w})$ in $1 \%$ intervals were prepared. In addition, 23 replicates evenly distributed from $5 \%$ to $55 \%(\mathrm{w} / \mathrm{w})$ sucrose were prepared giving a total of 75 samples. Water was purified using a Milli-Q ${ }^{\circledR}$ filter system. The sucrose used was BioUltra grade (>99.5\%, Sigma Aldrich, Darmstadt, Germany).

\section{Spectrometer setup}

The spectrometer setup is shown in Figure 1. The light source is a pulsed, supercontinuum laser source (SuperK COMPACT, NKT Photonics, Birkerød, Denmark) emitting 400-2500 nm pulses of approximately 1 ns duration and operated at $20 \mathrm{kHz}$ repetition rate. Its output is directed into $10 \mathrm{~km}$ of a custom dispersion compensating silica fibre (II micro, OFS Fitel, Norcross, Georgia, USA). The attenuation profile of the fibre results in loss of light outside the utilised $1100-1700 \mathrm{~nm}$ window because of increasing Rayleigh scattering at shorter wavelengths and a higher absorbance from silica at longer wavelengths. The fibre dispersion profile delays the propagation of shorter compared to that of longer wavelengths, resulting in stretching the 1 ns input pulse to an approximately 600 ns output pulse. The wavelengths towards $1700 \mathrm{~nm}$ travel faster through the dispersion compensating silica fibre compared to the shorter wavelengths towards $1100 \mathrm{~nm}$. The fibre output is collimated using an off-axis parabolic mirror (PM) (RC08PC-P01, Thorlabs, Mölndal, Sweden) and directed to a 50:50 beam splitter (BSW29, Thorlabs, Mölndal, Sweden). Of the two outgoing beams from the beamsplitter, one is directed onto the sample and then to an InGaAs detector (PDA10CF-EC, Thorlabs, Mölndal, Sweden). The other beam is measured by an identical second detector functioning as a reference. The light is split in two in order to correct for the pulse-to-pulse intensity variations in the supercontinuum generation process. ${ }^{10}$ A pulse-to-pulse normalisation is, therefore, performed by dividing the signal from the sample detector with the signal from the reference detector. The beamsplitter was set with a small angle (approximately $20^{\circ}$ ) between the incoming beam and the reference beam in order to avoid dependence on polarisation. ${ }^{11}$ Both analogue electrical signal outputs are captured in parallel by a digital oscilloscope (PicoScope 5444B, Picotech, Cambridgeshire, UK) with acquisition synchronised with the laser source's output trigger signal. In the spectrometer, each scan is the average signal of 2000 sample and reference pulse pairs. In order to improve the signal-to-noise ratio, 36 scans are averaged which gives a total of $36 \times 2000 \times 2=144,000$ pulses per spectrum. The total measurement time was $16 \mathrm{~s}$ per spectrum. Currently the spectrometer is measuring only every fourth pulse because of the oscilloscope's re-arm time. This means that spectra with the same signal-tonoise ratio could be acquired within $4 \mathrm{~s}$ by using an oscilloscope with a sufficiently fast re-arm time. Spectra are acquired, averaged and processed using in-house built Python-scripted software.

The transformation from a time-domain into a frequency-domain was done experimentally by measuring five narrow optical band pass filters. The optical filters had a centre wavelength accuracy $\pm 2-2.4 \mathrm{~nm}$ and a full width at half maximum (FWHM) accuracy of $\pm 10-12 \mathrm{~nm}$. The five filter measurements were fitted with a third-degree polynomial, as shown in Figure $1 \mathrm{~B}$, to achieve the coefficients for transformation from time to frequency domain. After transforming the data into the frequency domain, then the interval between measurement points is not equidistant. The spectral sampling resolution is $3.9 \mathrm{~nm}$ and $6.7 \mathrm{~nm}$ at $1700 \mathrm{~nm}$ and $1100 \mathrm{~nm}$, respectively.

The absorbance was calculated as $-\log _{10}\left(I_{S} / I_{0}\right)$ where $I_{0}$ is the light intensity with an empty cuvette in the sample holder and $I_{S}$ is the light intensity with a cuvette with sample inserted in the sample holder. The cuvette had a path length of $1 \mathrm{~mm}$. All samples were measured in random order.

\section{PCA and MCR}

In a PCA, ${ }^{12}$ the variance in a two-way dataset $\mathbf{X}$ with I rows $(|=1, \ldots|$,$) for samples and J$ columns $(j=1, \ldots, J)$ for variables can be decomposed into $F$ components $(f=1, \ldots, F)$ into the bi-linear model:

$$
\mathrm{X}=\mathrm{TP} \mathrm{P}^{\mathrm{T}}+\mathrm{E}
$$




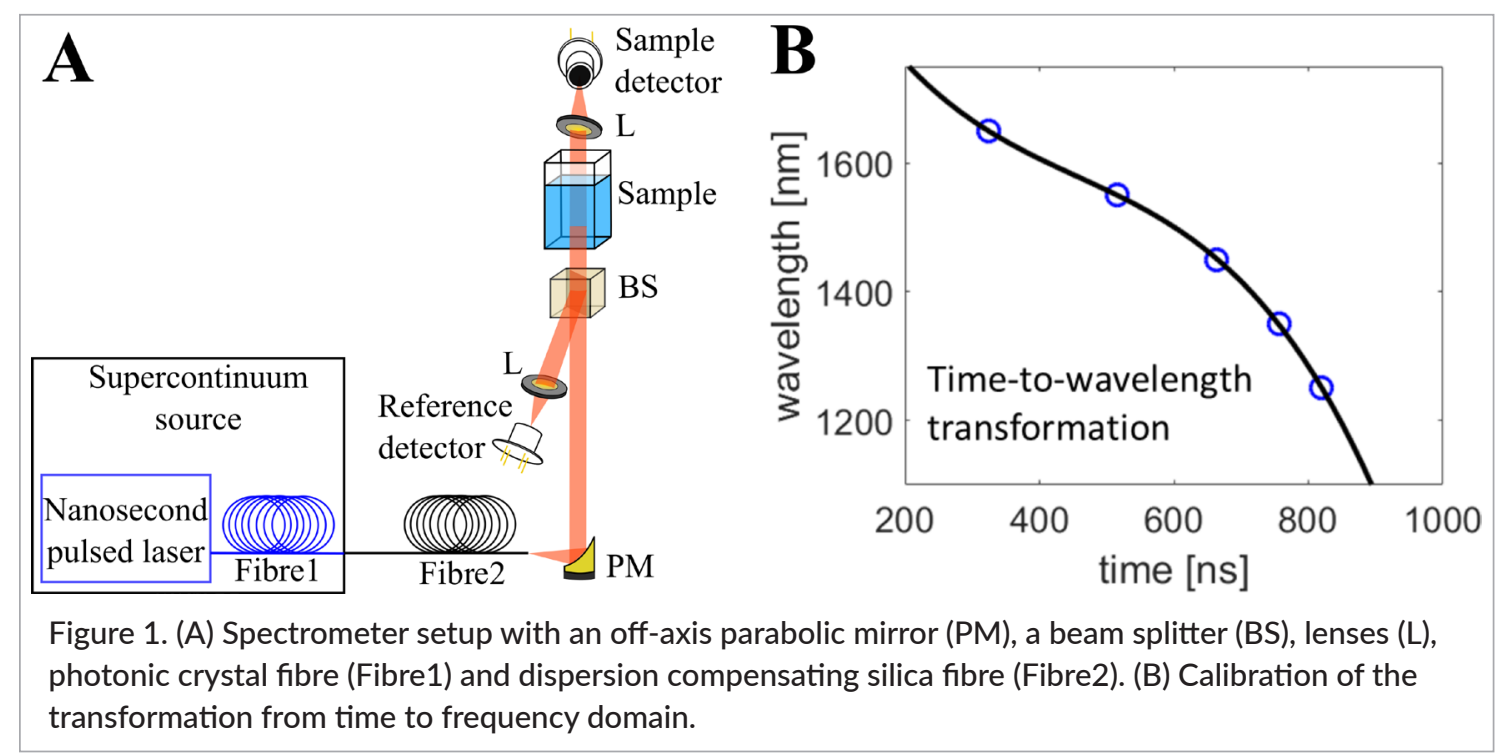

with the least-squares solution

$$
\min _{T, P} \sum_{i, j}\left\|x_{i j}-\sum_{f=1}^{F} t_{i f} p_{j f}\right\|^{2}
$$

where the sample scores $\mathrm{T}$ (size $/ \times F$ ) is the projection of variance onto the variable loadings $P($ size $J \times F)$ using $F$ components.

Self-modelling curve resolution or Multivariate Curve Resolution $(M C R)^{13,14}$ is similar to PCA in that it is a bilinear model used to decompose data into its structural parts. Where PCA decomposes the data into sequentially decreasing orders of variance, MCR fits F number of bilinear components simultaneously into a set of concentration profiles $\mathbf{C}$ (size $/ \times F$ ) and pure spectral profiles $\mathbf{S}$ $($ size $J \times F)$ for a dataset $X($ size $\mid \times J)$.

$$
\mathrm{X}=\mathrm{CS}^{\top}+\mathrm{E}
$$

Using alternating least squares ${ }^{15}$ (MCR-ALS) both concentration profiles (C) and pure spectral profiles (S) are optimised simultaneously in an iterative cycle. The challenges with MCR-ALS are its dependence on the initial guess of $\mathbf{S}$ or $\mathbf{C}$, its slow convergence, its dependence on independent variations amongst the constituents and its ambiguity in the solution. The strength of MCR-ALS is its capacity to resolve the pure underlying spectra and the ease with which different constraints can be applied. Imposing constraints can help in decreasing the risk of ambiguities and in this work a non-negativity constraint in the spectral mode have been used which is a realistic assumption for a chemical system observed by NIR spectroscopy. The MCR-ALS was initialised using random initial $\mathbf{S}$ and continued using a non-negative constraint on S. ALS iterations were performed until convergence was detected as the RMSE change dropping below 1e-12. The MCR model was applied to the spectral region $1151-1668 \mathrm{~nm}$.

\section{Data treatment}

Peak positions were investigated by calculating the Savitzky-Golay second derivative spectra ${ }^{16}$ (second degree polynomial, window size 9) in the PLS toolbox. Initial investigation of the dataset was conducted using PCA, and was carried out in the PLS toolbox 7.5 (Eigenvector Research, Inc., Manson, WA, USA) on mean centred spectral data. The MCR models were built using an in-house toolbox running in MATLAB R2015b (MathWorks Inc., Natick, MA, USA). Raw/non-processed spectra were used for MCR-ALS.

\section{Results}

The raw spectra in Figure 2A and the second derivative spectra in Figure 2B show that when the sucrose concentrations increase, the first overtone of $\mathrm{OH}$ stretching at about $1450 \mathrm{~nm}$ decreases, which is also the case for the $\mathrm{OH}$ combination band at $1151 \mathrm{~nm} .{ }^{17}$ In contrast, the band at about $1566 \mathrm{~nm}$, originating from intramolecularly hydrogen-bonded $\mathrm{OH}$ and the first overtone band at $1689 \mathrm{~nm}$, originating from $\mathrm{CH}$ stretching increase 


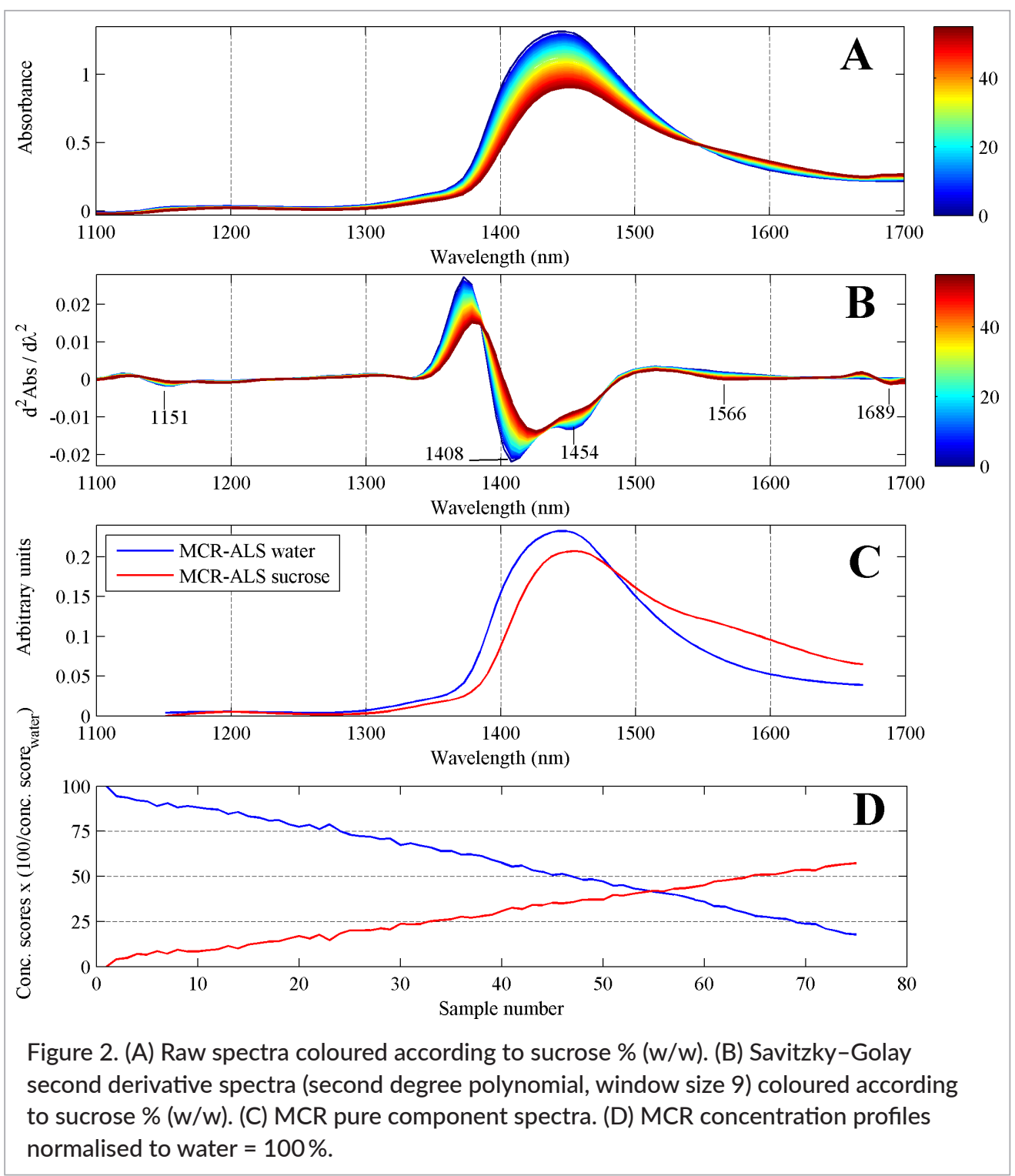

together with higher sucrose concentrations. ${ }^{17}$ In addition, the absorbance peak seen in the raw spectra at around $1450 \mathrm{~nm}$ and the valley in the second derivative spectra at $1408 \mathrm{~nm}$ shifts to longer wavelengths by the addition of more sucrose.

MCR was applied to the spectra in order to investigate the quality of the spectra and to study if it is possible to separate the spectral contribution from sucrose and water. Using PCA, it was determined that the system was sufficiently explained using two principal components, and thus is of rank two. The first two components showed significantly higher contributions which agrees with the fact that the chemical system is binary, i.e. contains only two ingredients. The two-component MCR solution consistently showed the same solution independent of the random initialisation. The resulting pure spectra and related concentration profile is shown in Figure 2, and the model explains $99.99 \%$ of the spectral variance. The first overtone $\mathrm{OH}$ stretching of the pure spectrum of sucrose $\left(\mathrm{S}_{\text {sucrose }}\right)$, represented by the red line in Figure $2 \mathrm{C}$, displays a shift towards longer wavelengths in peak maxima compared to the pure spectrum of water $\left(\mathbf{S}_{\text {water }}\right)$. This basically reflects that the sucrose hydroxyl groups are participating (on average) in more hydrogen bonds and that the donor-H bonding strength is weakened and thus the vibrational frequency is lowered (the wavelengths are increased). Furthermore, the pure spectrum of sucrose $\left(\mathrm{S}_{\text {sucrose }}\right)$ has a higher intensity from $1500 \mathrm{~nm}$ to $1668 \mathrm{~nm}$ compared to the pure spectrum of water. This is due to first overtones of the $\mathrm{CH}$ stretching vibra- 

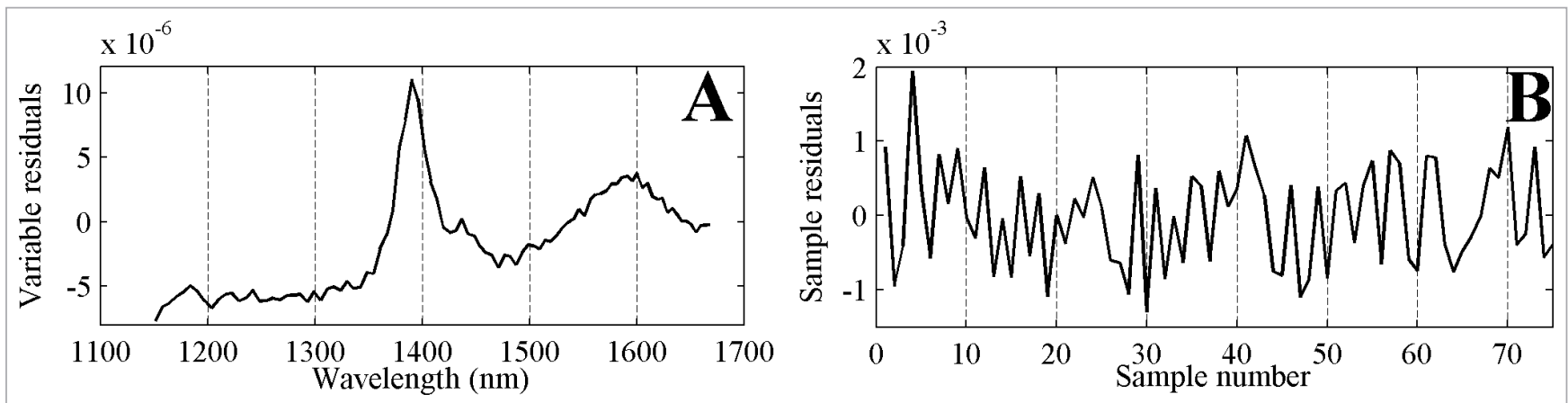

Figure 3. Mean residuals for the MCR model for (A) each wavelength and (B) each sample.

tions from the $\mathrm{CH}$ connected to primary and secondary hydroxyl groups in the sucrose structure. The concentration profile of sucrose $\left(\mathrm{C}_{\text {sucrose }}\right)$ in Figure $2 \mathrm{D}$ follows the expected change from $0 \%$ to $55 \%$ which strengthens the validity of the results. The residual $(\mathrm{E})$ for each variable and sample in Figure 3 shows what is not included in the MCR model. The random pattern of the sample residuals is interpreted as the uncertainties that are present in all experimental measurements. A more systematic pattern is seen in the variable residuals in Figure $3 \mathrm{~A}$ by a peak at $1400 \mathrm{~nm}$ and $1600 \mathrm{~nm}$. The peak at $1400 \mathrm{~nm}$ might be due to changes in temperature, ${ }^{18}$ since the early stage of this spectrometer did not include any temperature control. The peak at $1600 \mathrm{~nm}$ is exactly in the wavelength range of the $\mathrm{CH}$ stretching vibrations and might be interpreted as an interaction between water and sucrose that results in a very small contribution of $5 \times 10^{-6}$. A non-linear effect with changing sucrose concentration in water-sucrose solutions has previously been investigated with Raman ${ }^{19}$ and NIR $^{20}$ spectroscopy. The previous studies interpreted the non-linear behaviour as changes in the hydrogen bonding caused by sucrose behaving as a structure breaker at lower concentrations and as a structure maker at higher concentrations. In the studies by Mathlouthi et al. ${ }^{19}$ and Giangiacomo ${ }^{20}$ it was hypothesised that, at a certain sucrose percentage, there is a sudden change in the hydrogen bonding. This study measured samples with a much smaller interval of $1 \%(\mathrm{w} / \mathrm{w})$ sucrose concentrations compared to the two previous studies that used intervals of $5-10 \%$ sucrose $(\mathrm{w} / \mathrm{w})$. The first principal component (PC) from a PCA model showed a non-linear decrease when plotted against the sucrose \% $(\mathrm{w} / \mathrm{w})$ and the non-linear decrease was even clearer when plotted against the sucrose mole fraction \% (Figure 4). From Figure 4A it, therefore, seems likely that there is a non-linear interaction between water
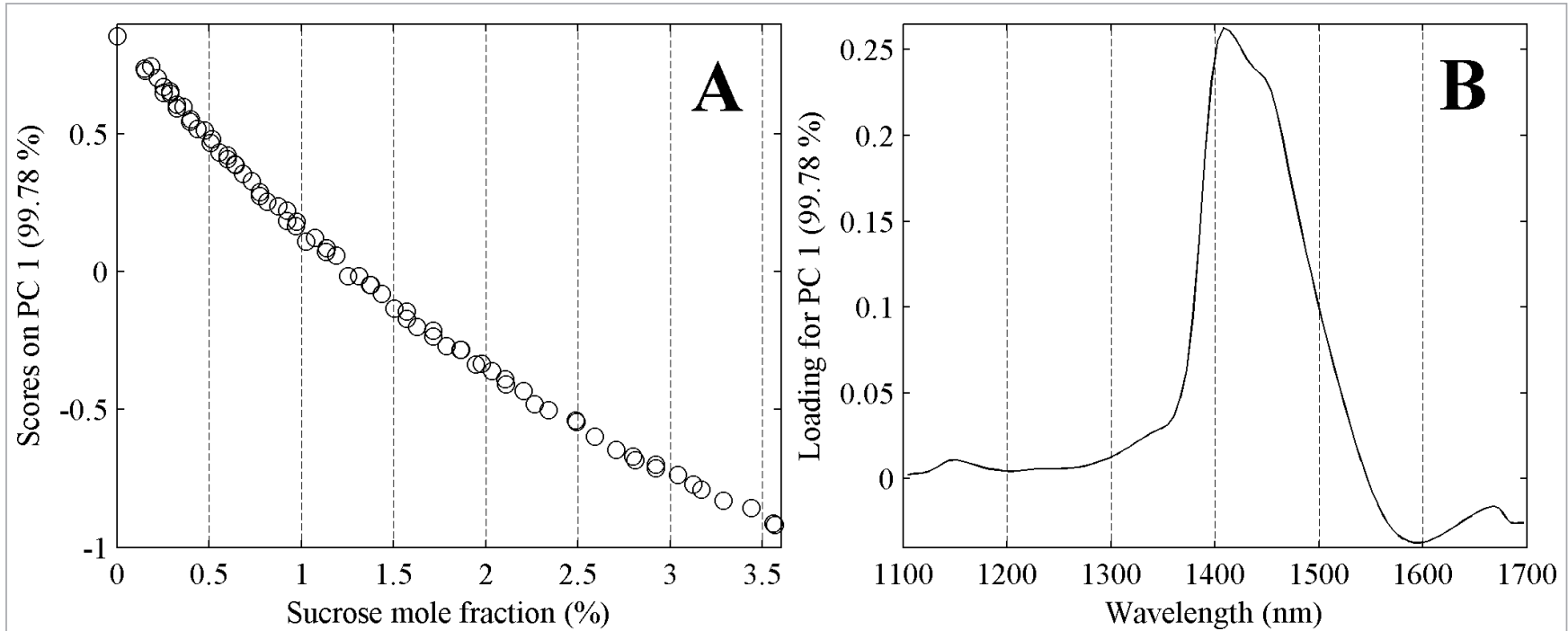

Figure 4. PCA model. (A) PC1 versus sucrose mole fraction. (B) PC1 loading. 
and sucrose and this might be the reason why the MCR model does not capture the residual at $1600 \mathrm{~nm}$.

The quality of spectra can be evaluated by the useful chemical information versus the noise. In this study, the chemical variance captured by the MCR model was used to determine the noise level which in this case was on the order of $99.99 \%$. Thus, just $0.01 \%$ noise was rejected by the model system.

\section{Outreach}

The use of a supercontinuum light source together with a wavelength separating fibre can provide a vibration insensitive and fast spectrometer. This paper shows the proof of principle and first results of the all-fibre instrument. The all-fibre instrument developed for this study has a spectral range from $1100 \mathrm{~nm}$ to $1700 \mathrm{~nm}$, which gives a spectral window (the so-called first overtone region) with information on all major food components, namely carbohydrates, fats, proteins and water. The spectrometer is still in a very early stage and many improvements, such as increasing the measurement speed, resolution and spectral range, may be possible in the near future. The potential of this spectrometer has been shown for a controlled laboratory experiment and more industrial tests have to be performed in order to appreciate the full potential of this new method.

\section{Acknowledgements}

The authors wish to acknowledge the generous financial support from The Danish National Advanced Technology Foundation (now Innovation Fund Denmark) to the project entitled "Light \& Food".

\section{References}

1. K. Goda and B. Jalali, "Dispersive Fourier transformation for fast continuous single-shot measurements", Nat. Photonics. 7, 102-112 (2013). https:// doi.org/10.1038/nphoton.2012.359

2. C. Lei, B.S. Guo, Z.Z. Cheng and K. Goda, "Optical time-stretch imaging: Principles and applications", Appl. Phys. Rev. 3, 011102 (2016). https://doi. org/10.1063/1.4941050
3. J.M. Dudley, G. Genty and S. Coen,

"Supercontinuum generation in photonic crystal fiber", Rev. Mod. Phys. 78, 1135-1184 (2006). https:// doi.org/10.1103/RevModPhys.78.1135

4. Y. Roggo, L. Duponchel and J.P. Huvenne, "Quality evaluation of sugar beet (Beta vulgaris) by near-infrared spectroscopy", J. Agric. Food Chem. 52, $1055-$ 1061 (2004). https://doi.org/10.1021/jf0347214

5. J.R. Santos, O. Viegas, R. Páscoa, I. Ferreira, A. Rangel and J.A. Lopes, "In-line monitoring of the coffee roasting process with near infrared spectroscopy: Measurement of sucrose and colour", Food Chem. 208, 103-110 (2016). https://doi. org/10.1016/j.foodchem.2016.03.114

6. P. Valderrama, J.W.B. Braga and R.J. Poppi, "Variable selection, outlier detection, and figures of merit estimation in a partial least-squares regression multivariate calibration model. A case study for the determination of quality parameters in the alcohol industry by near-infrared spectroscopy", J. Agric. Food Chem. 55, 8331-8338 (2007). https://doi.org/10.1021/ jf071538s

7. S. Grassi, C. Alamprese, V. Bono, E. Casiraghi and J.M. Amigo, "Modelling milk lactic acid fermentation using multivariate curve resolution-alternating least squares (MCR-ALS)", Food Bioproc. Technol. 7, 1819-1829 (2014). https://doi.org/10.1007/s11947013-1189-2

8. J.M. González-Sáiz, I. Esteban-Díez, S. RodríguezTecedor and C. Pizarro, "Valorization of onion waste and by-products: MCR-ALS applied to reveal the compositional profiles of alcoholic fermentations of onion juice monitored by near-infrared spectroscopy", Biotechnol. Bioeng. 101, 776-787 (2008). https://doi.org/10.1002/bit.21939

9. A. Blanco, A.C. Peinado and J. Mas, "Elucidating the composition profiles of alcoholic fermentations by use of ALS methodology", Anal. Chim. Acta. 544, 199-205 (2005). https://doi.org/10.1016/j. aca.2005.01.033

10. J. Ramsay, S. Dupont and S.R. Keiding, "Pulse-topulse noise reduction in infrared supercontinuum spectroscopy: polarization and amplitude fluctuations", Laser Phys. Lett. 11, 095702 (2014). https:// doi.org/10.1088/1612-2011/11/9/095702

11. T. Ringsted, S. Dupont, J. Ramsay, B.M. Jespersen, K.M. Sørensen, S.R. Keiding and S.B. Engelsen, "Near-infrared spectroscopy using a supercontinuum 
laser: application to long wavelength transmission spectra of barley endosperm and oil", Appl. Spectrosc. 70, 1176-1185 (2016). https://doi. org/10.1177/0003702816652361

12. H. Hotelling, "Analysis of a complex of statistical variables into principal components", J. Educ. Psychol. 24, 417-441 (1933). https://doi.org/10.1037/ h0071325

13. A. de Juan, J. Jaumot and R.A. Tauler, "Multivariate Curve Resolution (MCR). Solving the mixture analysis problem", Anal. Methods. 6, 4964-4976 (2014). https://doi.org/10.1039/c4ay00571f

14. W.H. Lawton and E.A. Sylvestre, "Self modeling curve resolution", Technometrics. 13, 617-633 (1971). https://doi.org/10.2307/1267173

15. J. De leeuw, F.W. Young and Y. Takane, "Additive structure in qualitative data - An alternating least squares method with optimal scaling features", Psychometrika 41, 471-503 (1976). https://doi. org/10.1007/BF02296971

16. A. Savitzky and M.J.E. Golay, "Smoothing and differentiation of data by simplified least squares procedures", Anal. Chem. 36, 1627-1639 (1964). https:// doi.org/10.1021/ac60214a047
17. L.G. Weyer and S.C. Lo, "Spectra-structure correlations in the near-infrared", in Handbook of Vibrational Spectroscopy, Ed by J.M. Chalmers and P.R. Griffiths. John Wiley \& Sons, Chichester, UK, Vol. 3, pp. 18171837 (2002). https://doi.org/10.1002/0470027320. s4102

18. B. Czarnik-Matusewicz, S. Pilorz and J.P. Hawranek, "Temperature-dependent water structural transitions examined by near-IR and mid-IR spectra analyzed by multivariate curve resolution and twodimensional correlation spectroscopy", Anal. Chim. Acta 544, 15-25 (2005). https://doi.org/10.1016/j. aca.2005.04.040

19. M. Mathlouthi, C. Luu, A.M. Meffroy-Biget and D.V. Luu, "Laser-Raman study of solute-solvent interactions in aqueous solutions of D-fructose, D-glucose, and sucrose", Carbohydr. Res. 81, 213-223 (1980). https://doi.org/10.1016/s0008-6215(00)85653-0

20. R. Giangiacomo, "Study of water-sugar interactions at increasing sugar concentration by NIR spectroscopy", Food Chem. 96, 371-379 (2006). https://doi. org/10.1016/j.foodchem.2005.02.051 